\title{
Tratamiento multidisciplinario de diente con pronóstico desfavorable. Reporte de caso.
}

\section{Multidisciplinary tooth treatment with unfavorable prognosis. Case report.}

\author{
Jaime Abarca ${ }^{1 *}, 2$, César Díaz ${ }^{1}$, Mario Garay¹, Héctor Monardes ${ }^{2,3}$
}

\author{
1. Facultad de Odontología, Universidad San \\ Sebastián, Sede Patagonia, Puerto Montt, Chile \\ 2. Postítulo de Especialización en Endodoncia, \\ Facultad de Odontología, Universidad San \\ Sebastián, Sede Santiago, Chile \\ 3. Facultad de Odontología, Universidad San \\ Sebastián, Sede Santiago, Chile \\ * Correspondencia autor. Jaime Abarca Reveco \\ | Facultad de Odontología Universidad San \\ Sebastián | Dirección: Lago Panguipulli 1390, \\ Pelluco Alto, Puerto Montt, Chile | Teléfono: \\ +56652325536 | E-mail: Jaime.abarca@uss.cl. \\ Trabajo recibido el 19/02/2018. \\ Aprobado para su publicación el 16/03/2018
}

\author{
RESUMEN \\ Las perforaciones radiculares son uno de los accidentes que pueden ocurrir dentro del \\ tratamiento endodóntico rehabilitador de un diente y comprometen el pronóstico de éste. \\ El objetivo de este reporte es describir el tratamiento y seguimiento de un diente con \\ pronóstico desfavorable, con falsa vía y perforación en el 1/3 medio radicular. El manejo \\ de la perforación se realizó con Biodentine ${ }^{\mathrm{TM}}$ y la rehabilitación con prótesis fija unitaria con \\ poste de fibra individualizado. A los controles clínicos y radiográficos de 6, 12 y 24 meses el \\ tratamiento se encontró asintomático y en función, pudiéndose considerar exitoso. \\ PALABRAS CLAVE: \\ Diente endodónticamente tratado, Perforación radicular, Biodentine ${ }^{\mathrm{TM}}$, Poste de Fibra. \\ Rev. Clin. Periodoncia Implantol. Rehabil. Oral Vol. 12(2); 87-89, 2019.

\begin{abstract}
A radicular perforation is a mishap that can occur during the Endodontic and Restorative Treatment of a tooth and it can compromise its short and long-term prognosis. The aim of this report is to describe the treatment and follow up of a tooth with an unfavorable prognosis that had a perforation in the radicular middle third. The perforation was repaired with Biodentine $\mathrm{T}^{\mathrm{TM}}$ and a fiber post and crown were placed. The patient had a follow up at 6,12 and 24 months and the tooth was found asymptomatic and functional, which can be considered a clinical success.

KEY WORDS:
\end{abstract} \\ Endodontically treated tooth, Root perforation, Biodentine ${ }^{\mathrm{TM}}$, Post and Core.
}

Rev. Clin. Periodoncia Implantol. Rehabil. Oral Vol. 12(2); 87-89, 2019

\section{INTRODUCCIÓN}

El tratamiento endodóntico posee un pronóstico favorable, mayor al $90 \%$, cuando no está asociado a periodontitis apical previa y está directamente relacionado al correcto sellado del canal radicular y a la calidad del tratamiento restaurador definitivo(1).

La perforación radicular es una comunicación mecánica, iatrogénica o patológica entre el sistema de canales radiculares y la superficie externa del diente. La causa de la perforación iatrogénica es a menudo el resultado de la desalineación de las fresas o de los instrumentos motorizados durante la preparación del acceso endodóntico o la preparación del canal radicular. Algunas se crean durante el tratamiento rehabilitador con prótesis fija unitaria, especialmente en la conformación del canal radicular para recibir el poste ${ }^{(2)}$.

Varios materiales han sido usados para reparación radicular, incluidos amalgama, óxido de zinc eugenol, hidróxido de calcio, resina compuesta y vidrio ionómeros. Sin embargo, ninguno de éstos son ideales para las condiciones y requerimientos de este tipo de tratamiento ${ }^{(3)}$. Un material ideal debiera ser biocompatible, dimensionalmente estable, insoluble, radiopaco, permitir una fácil manipulación y colocación, además de proveer un buen sellado ${ }^{(4)}$.

Biodentine $^{\mathrm{TM}}$ (Septodont, Saint Maur des Fosses, France) es un material a base de silicato de calcio de alta pureza, compuesto de silicato tricálcico, carbonato de calcio, óxido de zirconio y un líquido a base de agua que contiene cloruro de calcio como acelerador de fraguado y agente reductor. Biodentine ${ }^{\mathrm{TM}}$ se recomienda como sustituto de la dentina en restauraciones de resina compuesta y como material de reparación endodóntica debido a su buena capacidad de sellado, alta resistencia compresiva, tiempo de fraguado corto, biocompatibilidad, bioactividad y propiedades de biomineralización ${ }^{(5)}$.

El objetivo de este reporte es describir el manejo endodóntico y rehabilitador de un diente con pronóstico desfavorable debido a una perforación radicular.

\section{INFORME DEL CASO}

Paciente de género femenino de 60 años de edad, sin antecedentes mórbidos de importancia. Referido por fracaso en la localización del canal radicular al intentar desobturar para repetir tratamiento.

$\mathrm{Al}$ examen clínico intraoral se observa diente 2.1 con restauración coronaria provisoria, asintomático. Al examen radiográfico se observa falsa vía en relación a diente 2.1 (Figura 1).

Diagnóstico, Falsa vía en diente 2.1 previamente tratado.

Pronóstico, desfavorable.

Tratamiento, en una primera sesión se localiza el canal obturado y la falsa vía, que consiste en una perforación radicular hacia palatino (Figura 2), se procede a realizar la desobturación radicular con técnica mecanizada, preparación químico mecánica con sistema Reciproc R50 (VDW Germany), obturación del sistema de canales radiculares con 

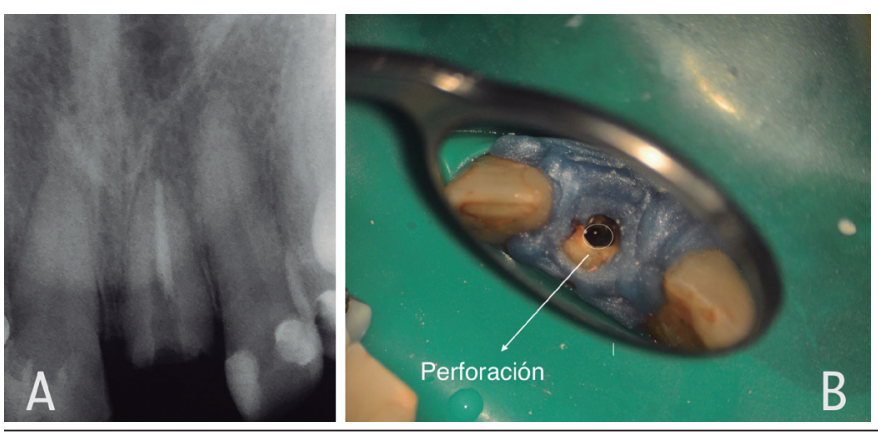

Figura 1. Radiografía preoperatoria con falsa vía diente 2.1. 1B. Situación clínica inical, se observa falsa vía en dirección palatina.
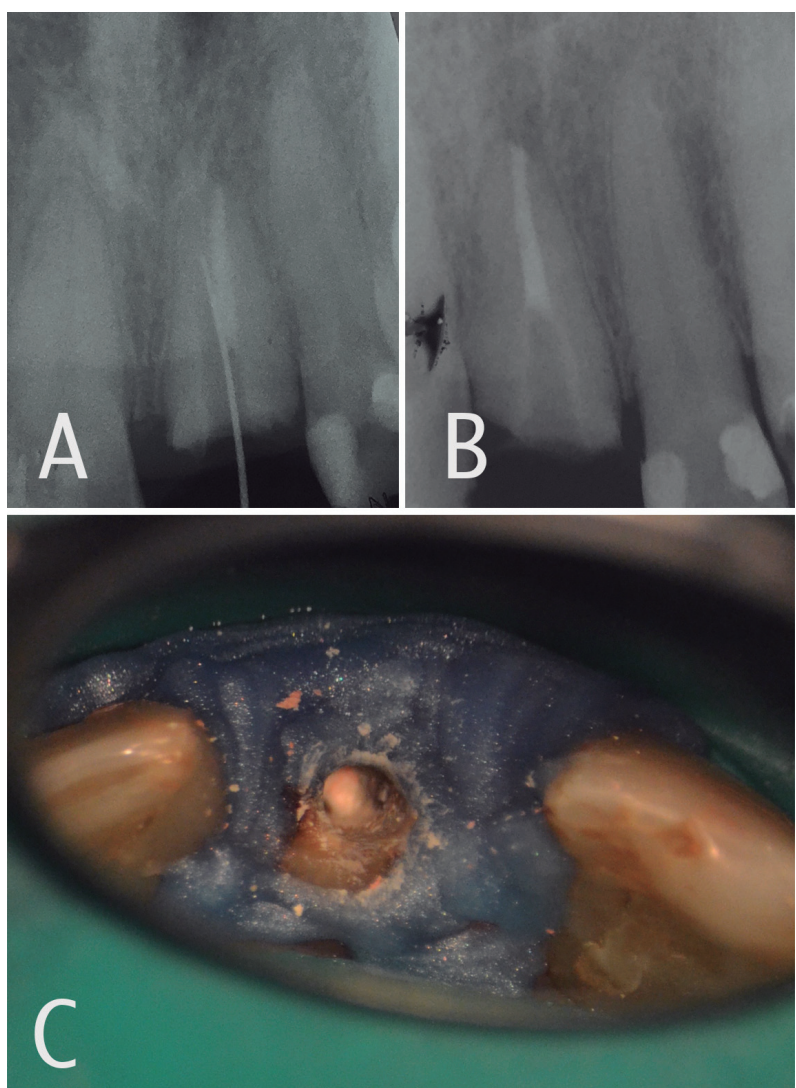

Figura 2. Radiografía con instrumento en falsa vía. 2B. Control obturación y sellado perforación y falsa vía con BIODENTINE. 2C. Imagen clínica de sellado de perforación y falsa vía diente 2.1.

compactación lateral y cemento TopSeal (Dentsply Sirona) y sellado de la falsa vía con Biodentine ${ }^{\mathrm{TM}}$ (Septodont, Saint Maur des Fosses, France) bajo magnificación con microscopio Zumax OMS 2300 (Figura 2).

En la fase rehabilitadora, se confecciona poste de fibra de vidrio individualizado, que consiste en un poste de fibra central ajustado íntimamente con resina compuesta a la anatomía interna del canal radicular (Figura 3). Cementación definitiva de poste con Relyx U200 (3 M ESPE ${ }^{\circledR}$, St. Paul, MN, EE. UU.). Se decidió observar la evolución del tratamiento por 4 meses mediante la restauración con una prótesis fija unitaria de carácter provisorio. Pasado este periodo se confeccionó una prótesis fija unitaria metal cerámica de carácter definitivo y se cementó con cemento resinoso Relyx U200 (3 M ESPE ${ }^{\circledR}$, St. Paul, MN, EE. UU.) (Figura 3).

En los controles clínicos y radiográficos de 6, 12 y 24 meses la paciente se encontraba asintomática y radiográficamente se observaron signos de salud periodontal pudiéndose catalogar de exitoso el tratamiento (Figura 4).

\section{DISCUSIÓN}

Las perforaciones dentarias ocurridas durante el tratamiento de canales o durante las maniobras previas al tratamiento rehabilitador reducen el pronóstico del tratamiento a un $54 \%-56 \%(2,6)$, siendo los
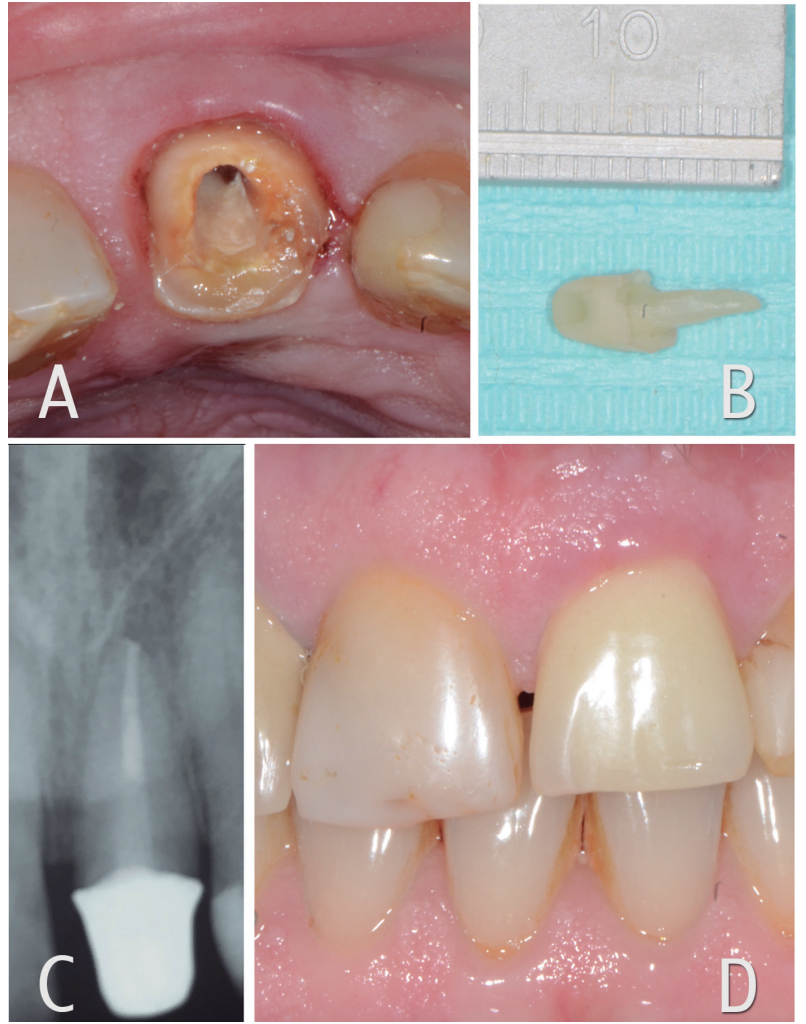

Figura 3. Imagen clínica remanente dentario y sellado de falsa vía. 3B. Poste de fibra individualizado. 3C. Control radiográfico 6 meses. 3D. Imágen clínica prótesis fija unitaria provisoria diente 2.1
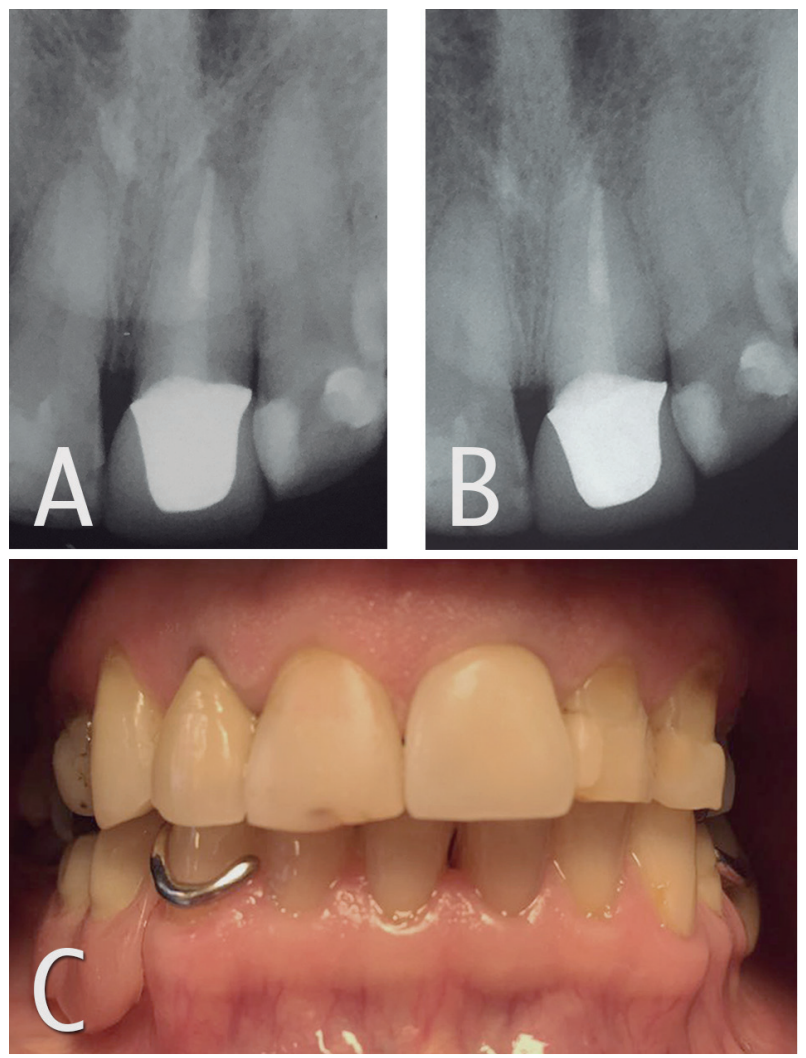

Figura 4. Control radiográfico 12 meses. 4B. Control radiográfico 24 meses. 4C. Situación clínica 24 meses de prótesis fija unitaria metal cerámica.

dientes maxilares los más afectados con un $74,5 \%$ de los casos, mientras que los mandibulares con un $25,5 \%^{(2,6)}$, pero los dientes maxilares tienen una mejor tasa de éxito que los dientes mandibulares, mostrando diferencias significativas $(P<0.05)^{(2)}$, lo que permite indicar con mayor 
seguridad los tratamientos de reparación de perforaciones en estos dientes.

Los factores que afectan el pronóstico de la perforación incluyen el tiempo trascurrido, la ubicación, el tamaño y la contaminación microbiana previa. La ubicación de la perforación es el factor pronóstico más crítico, pues las perforaciones en el tercio apical o medio de la raíz tienen un mejor pronóstico que las del tercio cervical o piso de la cámara ${ }^{(6)}$, al tener menor probabilidad de contaminación con microorganismos del medio oral. Además, la presencia de un área radiolúcida adyacente al sitio de la perforación tiene menor probabilidad de obtener éxito en la reparación, posterior al tratamiento $(P<0.05)^{(2)}$. En el caso que se presenta en este trabajo, el diente tratado presentaba una perforación radicular en el tercio medio, sin un área radiolúcida en el sitio adyacente a la perforación, lo que presume un mejor pronóstico.

Existen pocos estudios en la literatura que evalúan el resultado de los procedimientos clínicos, incluida la reparación de perforaciones, con periodos de evaluación de más de 1 o 2 años. Una revisión sistemática de la literatura relacionada con la reparación de perforaciones radiculares ha estimado que el porcentaje de éxito de los tratamientos no quirúrgicos para reparar perforaciones es de un $72,5 \%$ (IC $61.9 \%-81.0 \%)^{(2)}$, y un estudio clínico retrospectivo en 50 dientes tratados hasta hace 116 meses (promedio 37 meses) reportó como "dientes funcionales" un $94 \%$ de dientes tratados de manera no quirúrgica y mediante una combinación de tratamiento quirúrgico / no quirúrgico ${ }^{(6)}$, resultados que en cierta medida avalan el realizar intervenciones de este tipo.

El uso materiales biocerámicos en endodoncia otorga una nueva alternativa de tratamiento para estos casos de difícil manejo. Entre estos materiales, se ha reportado que MTA mejora la tasa de éxitos de estos tratamientos, alcanzado un $80,9 \%$ (IC $67,1 \%-89,8 \%$ ), pero sin constituir una diferencia significativa(2). Un estudio clínico retrospectivo sostiene que el uso de MTA para reparar perforaciones tiene un éxito importante, 33 casos exitosos de 36 realizados(6). Las potenciales ventajas de los materiales biocerámicos en endodoncia estaría relacionada con sus propiedades biológicas y físico-químicas, biocompatibilidad, no ser tóxicos, no sufrir contracción, ser químicamente estables en el entorno biológico, tener la capacidad de formar Hidroxiapatita y producir una adhesión entre la dentina y el material(7). En este caso se utilizó un biocerámico denominado BiodentineTM, que además de cumplir con las propiedades antes descritas, posee propiedades físicas similares a la dentina(8), no produce cambio de coloración en el diente(9), presenta facilidad en la manipulación, adecuado tiempo de trabajo y tiempo de fraguado final que lo hacen idóneo para este caso.

Para reafirmar el pronóstico de este caso, la rehabilitación se realizó con poste de fibra individualizado, debido a que la similitud en el módulo elástico entre el poste de fibra, el cemento de resina y la dentina se percibió como una ventaja para mejorar el rendimiento de las restauraciones. Sin embargo, si el poste no posee una anatomía similar a la del canal radicular, especialmente a nivel coronal, la capa de cemento de resina sería excesivamente gruesa y es probable que se formen burbujas dentro de ella. Una solución para superar este problema es incrementar el grosor del poste con resina compuesta. Este sistema de postes individualizados aumenta la adaptación del poste a las paredes de la raíz y reduce el espesor del cemento de resina(10).

\section{CONCLUSIÓN}

El caso reportado obtuvo un resultado exitoso al cabo de dos años de seguimiento, a pesar de presentar un pronóstico dudoso de manera previa, debido a un trabajo multidisciplinario y a la utilización de técnicas y materiales actuales que ayudan a lograr mejores resultados clínicos y que conservan el órgano dentario.

\section{CONFLICTO DE INTERÉS}

Los Autores declaran no presentar conflicto de interés.

\section{RELEVANCIA CLÍNICA}

Reporte de caso con el uso de material Biocerámicos para tratar falsa vía con perforación y poste de fibra individualizado para la rehabilitación de diente con pronóstico desfavorable.

\section{Bibliografía}

1. Monardes H, Lolas C, Aravena J, González H, Abarca J. Evaluación del tratamiento endodóntico y su relación con el tipo y la calidad de la restauración definitiva. Rev Clin Periodoncia Implantol Rehabil Oral. 2016;9(2):108-13.

2. Siew K, Lee AH, Cheung GS. Treatment outcome of repaired root perforation: A systematic review and meta-analysis. J Endod. 2015;41(11):1795-804.

3. Zhou HM, Shen Y, Wang ZJ, Li L, Zheng YF, Hakkinen L, et al. In vitro cytotoxicity evaluation of a novel root repair material. J Endod. 2013;39(4):478-83.

4. Aggarwal V, Singla M, Miglani S, Kohli S. Comparative evaluation of push-out bond strength of ProRoot MTA, Biodentine, and MTA Plus in furcation perforation repair. $J$ Conserv Dent. 2013;16(5):462-5.

5. Guneser MB, Akbulut MB, Eldeniz AU. Effect of various endodontic irrigants on the push-out bond strength of biodentine and conventional root perforation repair materials. J Endod. 2013;39(3):380-4
6. Pontius V, Pontius O, Braun A, Frankenberger R, Roggendorf MJ. Retrospective evaluation of perforation repairs in 6 private practices. J Endod. 2013;39(11):1346-58 7. Wang Z. Bioceramic materials in endodontics. Endod Topics. 2015;32(1):3-30 8. Butt N, Talwar S, Chaudhry S, Nawal RR, Yadav S, Bali A. Comparison of physical and mechanical properties of mineral trioxide aggregate and Biodentine. Indian J Dent Res. 2014;25(6):692-7.

9. Valles M, Roig M, Duran-Sindreu F, Martinez S, Mercade M. Color stability of teeth restored with Biodentine: A 6-month in vitro study. J Endod. 2015;41(7):1157-60.

10. Clavijo VG, Reis JM, Kabbach W, Silva AL, Oliveira Junior OB, Andrade MF. Fracture strength of flared bovine roots restored with different intraradicular posts. J Appl Oral Sci. 2009;17(6):574-8 